\title{
Using Solubility Parameter Analysis to Understand Delignification of Poplar and Rice Straw with Catalyzed Organosolv Fractionation Processes
}

\author{
Fangchao Cheng, a,b,* Tulong Ouyang, ${ }^{\text {J Jianping Sun, }}$, Tao Jiang, ${ }^{\mathrm{a}}$ and Jianju Luo ${ }^{\mathrm{a}}$
}

\begin{abstract}
Biomass fractionation is important for the further conversion of the cellulosic fraction and the effective utilization of the lignin fraction for the production of biofuels and value-added products. Solvent properties play a crucial role on the fractionation efficiency of lignocellulosics using an organosolv fractionation process catalyzed by acidic ionic liquids (AILs). Herein, 12 organic solvents were selected as co-solvents with water based on their solubility parameters for lignin dissolution, including alcohols, alcohol ethers, lactones, and alkanolamines, in order to fractionate poplar and rice straw. Effects of fractionation liquor solubility parameters on the delignification efficiency of organosolv fractionation were investigated under the same conditions. Relative energy differences (REDs) of the cosolvent systems for lignin dissolution were closely related to the degree of delignification with adjusted coefficients of determination of 0.899 and 0.800 for poplar and rice straw processes, respectively. The application of solubility parameter analysis may provide a rational way to screen solvent or co-solvent fractionation systems for biomass fractionation process.
\end{abstract}

Keywords: Organosolv fractionation; Organic solvents; Delignification; Solubility parameters

Contact information: a: Guangxi Key Laboratory of Processing for Nonferrous Metallic and Featured Materials, School of Resources, Environment and Materials, Guangxi University, Nanning 530004, China; b: School of Materials Science and Engineering, Central South University of Forestry and Technology, Changsha 410004, China; *Corresponding author: fangchaocheng@gxu.edu.cn

\section{INTRODUCTION}

The organosolv fractionation process has been recognized as one of the most promising techniques for biomass fractionation or pretreatment due to its low environment impact, high delignification efficiency, and diverse products (Zhu et al. 2010). It provides an integrated pathway for the utilization of lignocellulosic biomass by producing highyield cellulose materials, organosolv lignin, and other chemicals, such as sugars and other by-products (Amiri et al. 2014). Organosolv fractionation can decrease the recalcitrance of biomasses by converting most of hemicelluloses and removing a large portion of lignin. Elevated enzymatic hydrolysis efficiency of cellulose materials can be received after the organosolv process (Santo et al. 2018). Thus, organosolv fractionation has been employed as the initial fractionation stage for biorefineries producing biofuels from lignocellulosics using the enzymatic hydrolysis-fermentation process (Romaní et al. 2011).

Delignification efficiency is crucial for the organosolv fractionation process to yield high-quality cellulose and valuable lignin raw materials (Schrems et al. 2012). Acidic catalysts are usually used during organosolv fractionation, which promote the cleavage of $\beta$-aryl ether bonds (e.g. $\beta$-O-4) via acidolysis or homolytic cleavage; breakage of these bonds leads to enhanced delignification of the biomass (Kangas et al. 2015). The cleavage 
of $\beta$-aryl ether bonds decreases the molecular weight of the native lignin, which improves its solubility in the organic solvent fractionation medium (Zhao et al. 2009; Pandey and Kim 2011). Thus, not only different physical (e.g. boiling point) and chemical properties (i.e. reactions with biomass components) but also differences on lignin solubility of the cosolvent systems will cause a great difference on delignification efficiency during organosolv fractionation processes (Zhao et al. 2009; Lv et al. 2013). Besides, the type of organic solvent also greatly influences the selectivity of lignin extraction during organosolv fractionation process (Oliet et al. 2002).

The selection of organic solvent will directly influence the fractionation efficiency of an organosolv fractionation process. Solubility parameters of solvents, which have been developed to predict polymer solubility in a medium, can provide a guide to selecting a solvent or medium for organosolv fractionation (Quang et al. 2016). The concept of the Hildebrand solubility parameter ( $\delta$-value) has been applied to explain the effect of different organic solvent concentrations in a solvent-water medium used in organosolv fractionation of hydrolyzed almond shells without a catalyst (Quesada-Medina et al. 2010). It has been revealed that the closer the fractionation medium's $\delta$-value to that of lignin, the greater the degree of delignification was obtained.

The effects of organic solvent concentrations on lignin removal during lignin extraction process of enzymatically hydrolyzed cornstalks without additional catalyst were also investigated with the Hildebrand solubility parameter ( $\delta$-value) (Ye et al. 2014). The concentration of organic solvent in the fractionation media that led to the highest delignification was correlated to the predictions using solubility parameter analysis. Recently, fractionation of lignocellulosic biomasses with organosolv fractionation has been studied by Cheng et al. (2018), who combined a series of aqueous high-boiling-point polyols solutions with acidic ionic liquids (AIL).

The solvent selection was investigated through analyzing the effects of lignin chemical structures and the Hansen solubility parameters of alcohols on the fractionation efficiency, and both chemical structures and solubility parameters of solvents obviously influenced the component yields and delignification rates. Although great efforts have been made to optimize organosolv fractionation through screening the promising organic solvents, the effects of solubility parameters of solvent systems on the organosolv fractionation efficiency have not been extensively studied (Zhao et al. 2009; Li et al. 2016). Especially, few studies focused on the selection of solvents for the organosolv fractionation with Hansen solubility parameters.

Accordingly, 12 organic solvents, including alcohols, alcohol ethers, lactones, and alkanolamines, were selected according to their solubility parameters for lignin dissolution. Several organic solvents, e.g. ethylene glycol monomethyl ether, ethylene glycol monoethyl ether, diethylene glycol monoethyl ether, diethylene glycol monomethyl ether, and $\gamma$-butyrolactone, were selected for use in the organosolv fractionation process for the first time. Rice straw and poplar biomasses were fractionated into cellulose-rich materials (CRM) and organosolv lignin using fractionation media comprised of organic solvent and water mixtures, along with AIL. Effects of fractionation media solubility parameters on delignification efficiency were investigated by conducting organosolv fractionation at the same conditions. These analyses on effects of solubility parameters of co-solvent systems on the delignification efficiency provided a rational and meaningful way to predict and screen solvent for the organosolv fractionation process. 


\section{EXPERIMENTAL}

\section{Materials}

Rice straw was cut and then pulverized using a lab mill (Model FZ102,Tianjin, China); the resulting powder was collected and sieved through 60 to 100 mesh screens. The ground powder was dried in an oven at $90{ }^{\circ} \mathrm{C}$ overnight and then extracted with acetone in a Soxhlet extractor for $6 \mathrm{~h}$. The extracted powder was dried again under the same condition. Poplar wood (Populous tomentosa Carr.) powder of 60 to 100 mesh was obtained with the same method.

1-Butyl-3-methylimidazolium hydrosulfate ([Bmim] $\left.\left[\mathrm{HSO}_{4}\right]\right)$ was purchased from the Lanzhou Institute of Chemical Physics of the Chinese Academy of Sciences. Ethanol, ethylene glycol and 1,2-propylene glycol were obtained from Tianjin Fuyu Fine Chemical Co., Ltd., China. $\gamma$-Butyrolactone, ethylene glycol monomethyl ether, ethylene glycol monoethyl ether, ethanolamine, diethylene glycol monomethyl ether, diethylene glycol monoethyl ether, diethylene glycol, 1,4-butanediol, and 1,3-butanediol were obtained from Shanghai Aladdin Bio-Chem Technology Co., Ltd., China. All other chemical reagents were purchased from Shanghai Aladdin Bio-Chem Technology Co., Ltd., China; these reagents were used as received.

\section{Biomass Fractionation Process (Organosolv fractionation)}

In a typical run, $30 \mathrm{~mL}$ of aqueous organic solvent medium $(24 \mathrm{~mL}$ organic solvent and $6 \mathrm{~mL}$ water), $1 \mathrm{~g}$ of 1-butyl-3-methylimidazolium hydrogen sulfate ([Bmim $\left.{ }^{+}\right]\left[\mathrm{HSO}_{4}{ }^{-}\right.$ ]), and $1 \mathrm{~g}$ of biomass powder were added to a $50 \mathrm{~mL}$ stainless steel autoclave; the contents were mixed thoroughly using a magnetic stirrer in order to remove entrained gas bubbles. Subsequently, the reactor was sealed, and the system was purged of air with argon gas three times. The reactor was then immersed in an oil bath at $200{ }^{\circ} \mathrm{C}$. It took $20 \mathrm{~min}$ for the reaction mixtures in the reactor to reach $200{ }^{\circ} \mathrm{C}$. Subsequently, the reactor was kept in the oil bath for another $10 \mathrm{~min}$, and the total heating time was $30 \mathrm{~min}$. Afterwards, the reactor was removed and allowed to cool to room temperature. The resulting solution was filtered through a nylon membrane $(0.4 \mu \mathrm{m})$ under vacuum in order to obtain CRM. The separated CRM solids were washed three times with $50 \mathrm{~mL}$ distilled water, and then dried. Distilled water $(100 \mathrm{~mL})$ was added to the combined filtrate to precipitate the lignin. Precipitated lignin was separated from the supernatant solution by vacuum filtration; it was washed with water three times.

CRM yield and lignin yield were calculated as the mass percentage of the recovered material to the mass of the original biomass. Mass loss was defined as the percentage difference in mass between the original biomass from the recovered yields of CRM and lignin as defined in Eq. 1:

$$
\text { Mass Loss }(\%)=100-\text { CRM Yield }(\%) \text { - Lignin Yield }(\%)
$$

The delignification rate represents the reduction of the lignin in CRM compared to the lignin in the original biomass,

$$
\text { Delignification Rate }(\%)=\frac{m_{B} \times L B-m_{c} \times L C}{m_{B} \times L B} \times 100 \%
$$

where $m_{B}$ and $m_{C}$ stand for the weight of original biomass and corresponding CRM. 


\section{Solubility Parameters Calculation}

The solubility parameter ( $\delta$-value) was first introduced by Hildebrand and Scott (1950). The parameter is defined as,

$$
\delta=(E / V)^{1 / 2}
$$

where $E$ is the energy of vaporization at zero pressure and $V$ is the molar volume. The polar force between molecules is not taken into account in the total solubility parameter theory, and the three dimensional solubility theory was developed by Hansen (2007). In this theory, the total energy of vaporization of a liquid arises from dispersion forces, permanent dipolepermanent dipole forces, and hydrogen bonding. The difference in solubility parameters between a solute and a solvent, which can reflect the capacity of the solvent to dissolve the solute, is expressed as $R_{a}$, given by the relation

$$
\left(R_{a}\right)^{2}=4\left(\delta_{\mathrm{D} 2}-\delta_{\mathrm{D} 1}\right)^{2}+\left(\delta_{\mathrm{P} 2}-\delta_{\mathrm{P} 1}\right)^{2}+\left(\delta_{\mathrm{H} 2}-\delta_{\mathrm{H} 1}\right)^{2}
$$

where $\delta_{\mathrm{D}}, \delta_{\mathrm{P}}$, and $\delta_{\mathrm{H}}$ are the solubility parameters contributed by dispersion, dipole-dipole forces, and hydrogen bonding, respectively; the subscript indices 1 and 2 represent the solvent medium and to the solute, respectively. If $R_{a}$ is less than the experimentallydetermined radius, $R_{o}$, of the solubility sphere for the solute itself, i.e. the relative energy difference $(\mathrm{RED})=R_{a} / R_{O}<1$, then the solute can be dissolved in the solvent medium. In the present study, aqueous solutions of organic solvents were employed as the organosolv fractionation medium to fractionate biomass; the $\delta$-values of the various media are calculated using (Barton 1975),

$$
\delta_{\mathrm{M}}=\varphi_{1} \delta_{1}+\varphi_{2} \delta_{2}
$$

where $\delta_{\mathrm{M}}$ is the $\delta$-value of the binary mixture, $\varphi$ is the volume fraction of the organic solvent (index 1) or water (index 2), and $\delta$ is the $\delta$-value of the organic solvent (index 1 ) or water (index 2).

The solubility parameters of aqueous organic solvent media for lignin dissolution (Table 1) were calculated with Eqs. 4 and 5 using values obtained from literature (Hansen 2007; Benazzouz et al. 2013). The solubility parameters of the solutes were experimentally determined by investigating the dissolution of milled wood lignin (MWL) as described in by Hansen (2007). Especially, it should be noted that MWL obtained by the fine grinding and facile extraction has been considered to reserve the original structure of lignin to a great extent since the mild separation process. Consequently, the solubility parameters of MWL should be able to relatively better reflect the solubility properties of protolignin than lignin from other sources.

\section{Chemical Composition Analysis}

The chemical composition of the original biomass and the CRM were determined by a combined method previously reported (Datta 1981; Cheng et al. 2014). Briefly, $0.1 \mathrm{~g}$ of biomass was combined with $30 \mathrm{~mL} 0.5 \mathrm{M} \mathrm{H}_{2} \mathrm{SO}_{4}$ solution, and the mixture was refluxed for $2 \mathrm{~h}$. The hemicellulose content was obtained by determining the reduction of biomass mass (after drying) from this treatment versus the original mass. The above dried residue was further treated with $2 \mathrm{~mL} 72 \mathrm{wt} \% \mathrm{H}_{2} \mathrm{SO}_{4}$ for $2 \mathrm{~h}$ at room temperature. Afterwards, the mixture was diluted with water to $3 \mathrm{wt} \% \mathrm{H}_{2} \mathrm{SO}_{4}$ and refluxed for $4 \mathrm{~h}$. The mass of the acid insoluble lignin from this treatment was determined after its drying; the amount of acid soluble lignin was then determined from the hydrolysis supernatant by UV-Vis absorption 
at $205 \mathrm{~nm}$ (Dence 1992). The cellulose content was calculated by difference after the determination of hemicellulose and lignin content values.

Ash content of original biomass was measured through calcining it in air at $575 \pm 25^{\circ} \mathrm{C}$ for $4 \mathrm{~h}$ and weighing the residual ash (GB/T 742-2008, 2008). The ash contents for poplar and rice straw were $0.8 \%$ and $11.3 \%$, respectively. The composition analysis on poplar was conducted without considering ash due to the limited ash content, while the composition determination of rice straw and the corresponding CRM need to subtract the ash content in each step.

Table 1. The Calculated Solubility Parameters of Aqueous Solutions of Selected Organic Solvents Used as Co-Solvents for the Organosolv fractionation Medium

\begin{tabular}{|c|c|c|c|c|}
\hline $\begin{array}{l}\text { Aqueous Media of Selected Organic Solvents } \\
\text { (Organic solvent:water of } 4: 1(\mathrm{v} / \mathrm{v}) \text { ) }\end{array}$ & $\begin{array}{c}\delta \mathrm{DM}^{*} \\
\left(\mathrm{MPa}^{1 / 2}\right)\end{array}$ & $\begin{array}{c}\delta \mathrm{PM}^{*} \\
\left(\mathrm{MPa}^{1 / 2}\right)\end{array}$ & $\begin{array}{c}\delta_{\mathrm{HM}^{*}} \\
\left(\mathrm{MPa}^{1 / 2}\right)\end{array}$ & $R E D_{\mathrm{ML}^{\#}}$ \\
\hline Ethanolamine/Water & 16.6 & 14.0 & 19.5 & 0.794 \\
\hline$\gamma$-Butyrolactone/Water & 18.2 & 14.8 & 8.4 & 0.820 \\
\hline 1,4-Butanediol/Water & 16.3 & 13.8 & 19.9 & 0.846 \\
\hline Diethylene Glycol/Water & 16.3 & 11.2 & 19.1 & 0.860 \\
\hline 1,3-Butanediol/Water & 16.3 & 9.6 & 19.7 & 0.906 \\
\hline 1,2-Propylene Glycol/Water & 16.5 & 9.1 & 21.2 & 0.928 \\
\hline Ethylene Glycol/Water & 16.6 & 10.4 & 23.3 & 0.942 \\
\hline Ethylene Glycol Monomethyl Ether/Water & 16.0 & 9.0 & 15.6 & 0.948 \\
\hline Ethylene Glycol Monoethyl Ether/Water & 16.0 & 8.9 & 14.0 & 0.967 \\
\hline Ethanol/Water & 15.7 & 8.6 & 18.0 & 0.999 \\
\hline Diethylene Glycol Monoethyl Ether/Water & 15.9 & 8.9 & 12.3 & 1.012 \\
\hline Diethylene Glycol Monomethyl Ether/Water & 16.0 & 7.8 & 12.6 & 1.028 \\
\hline \multicolumn{5}{|c|}{$\begin{array}{l}{ }^{*} \delta_{\mathrm{DM}}, \delta_{\mathrm{PM}}, \text { and } \delta_{\mathrm{HM}} \text { are the solubility parameters of organic solvent-water medium contributed by } \\
\text { dispersion, dipole-dipole forces, and hydrogen bonding, respectively. } \\
{ }^{*} R E D_{\mathrm{ML}} \text { is relative energy difference between organic solvent-water medium and lignin. }\end{array}$} \\
\hline
\end{tabular}

\section{RESULTS AND DISCUSSION}

The present study attempted to understand the effects of solubility properties of solvent fractionation media on biomass fractionation by AIL-catalyzed organosolv fractionation using high-boiling-point solvents; a solubility parameter analysis was performed. Twelve organic solvents that might dissolve lignin, which included alcohols, alcohol ethers, lactones, and alkanolamines, were selected according to their solubility parameters for lignin dissolution. (i. e., RED values of these solvents for lignin dissolution are less than 1 or around 1 (Hansen 2007)). All of these fractionation media were tested under the same conditions during organosolv fractionation to investigate the effects of the media's solubility parameters on biomass delignification. Fractionation parameters (temperature of $200{ }^{\circ} \mathrm{C}$, treatment time of $30 \mathrm{~min}$, and fractionation medium-to-biomass ratio of 4:1) were selected based on a previous organosolv fractionation study (Cheng et al. 2018).

Poplar is a fast-growing wood in Northern China, which has high productivity $(\mathrm{Hu}$ et al. 2015). It provides an important, sustainable, and renewable source for the production of biofuels and chemicals (Cantarella et al. 2004). Rice straw, as one of the most abundant agriculture wastes, was also considered as a promising biomass feedstock for economic 
and sustainable production of liquid biofuels and derivative chemicals (Hou et al. 2018). Herein, poplar and rice straw were examined as the woody and herbaceous biomass for the AIL-catalyzed organosolv fractionation. Aimed to analyze the influence of solvent type on the relationship between solubility parameters and delignification efficiency, different types of solvents were selected to fractionate poplar and rice straw, respectively. Six organic solvents, including alcohols and alcohol ethers, were selected to fractionate poplar. Afterwards, rice straw was treated using eight organic solvents, which included alcohols, alcohol ethers, lactones, and alkanolamines. Effects of RED values of these aqueous solvent media for lignin dissolution efficiencies were studied to understand the lignin solubility-delignification relationship of the organosolv fractionation process.

\section{Biomass Fractionation Analysis}

Fractionation results from the organosolv fractionation of poplar and rice straw using different fractionation media are summarized in Fig. 1. These co-solvent systems resulted in comparable CRM and lignin yields while there were varied delignification efficiencies during the processes of poplar and rice straw. With respect to the poplar, a delignification rate of more than $90 \%$ can be obtained with all the fractionation media examined. However, delignification rate of rice straw ranged from 70 to $93 \%$. According to Ouyang et al. (2018), organosolv fractionation using high-boiling-point alcohols and AIL can selectively delignify poplar and partially degrade lignin during the process. Similar results were observed for the organosolv fractionation using various aqueous solvent media including alcohols and other organic solvents. Rice straw contained higher silica content than other biomasses, which increased its recalcitrance to pretreatment, fractionation and hydrolysis (Alok et al. 2018). Silica is inert to acids (except hydrofluoric acid), while it is easy to react with alkali (Haysom and Ostatek-Boczynski, 2006; Jahan et al. 2015). It is reported that the extent of silica removal depends upon the alkalinity during the pretreatment process with rice straw; $1 \%$ sodium hydroxide has been found to remove up to nearly $62.2 \%$ of silica in rice straw (Sun et al. 2000). Hence, the present organosolv system catalyzed by AIL demonstrated lower delignification efficiency with rice straw than with poplar. The most alkaline solvent media, ethanolamine, exhibited the highest delignification efficiency and the highest CRM yield among the selected solvent-water systems examined for rice straw pretreatment. Compared with the rice straw processes, higher delignification selectivity can be obtained during the poplar processes.

Six solvents, including two alcohols (1,4-butanediol and ethylene glycol) and four alcohol ethers (diethylene glycol, ethylene glycol monomethyl ether, diethylene glycol monoethyl ether, and diethylene glycol monomethyl ether), were examined as organic solvent to fractionate poplar, along with water. The selected alcohol ethers afforded comparable component yields and delignification rates when compared to the two alcohols. The process using diethylene glycol afforded a lower CRM yield and a higher mass loss than the other fractionation media examined. This resulted in high cellulose purity and low amounts of hemicelluloses in the CRM. The process using its derivative, diethylene glycol monomethyl ether, showed high fractionation efficiency and led to higher CRM yield and less mass loss. To the best knowledge, ethylene glycol monomethyl ether, diethylene glycol monoethyl ether, and diethylene glycol monomethyl ether were investigated as co-solvents in organosolv fractionation processes for the first time. And these solvents can be produced from lignocellulose conversion, which are of sustainability (Xi et al. 2014). Considering their sustainable characteristics and effectiveness for biomass fractionation, these organic solvents can be regarded as effective co-media for organosolv fractionation. 

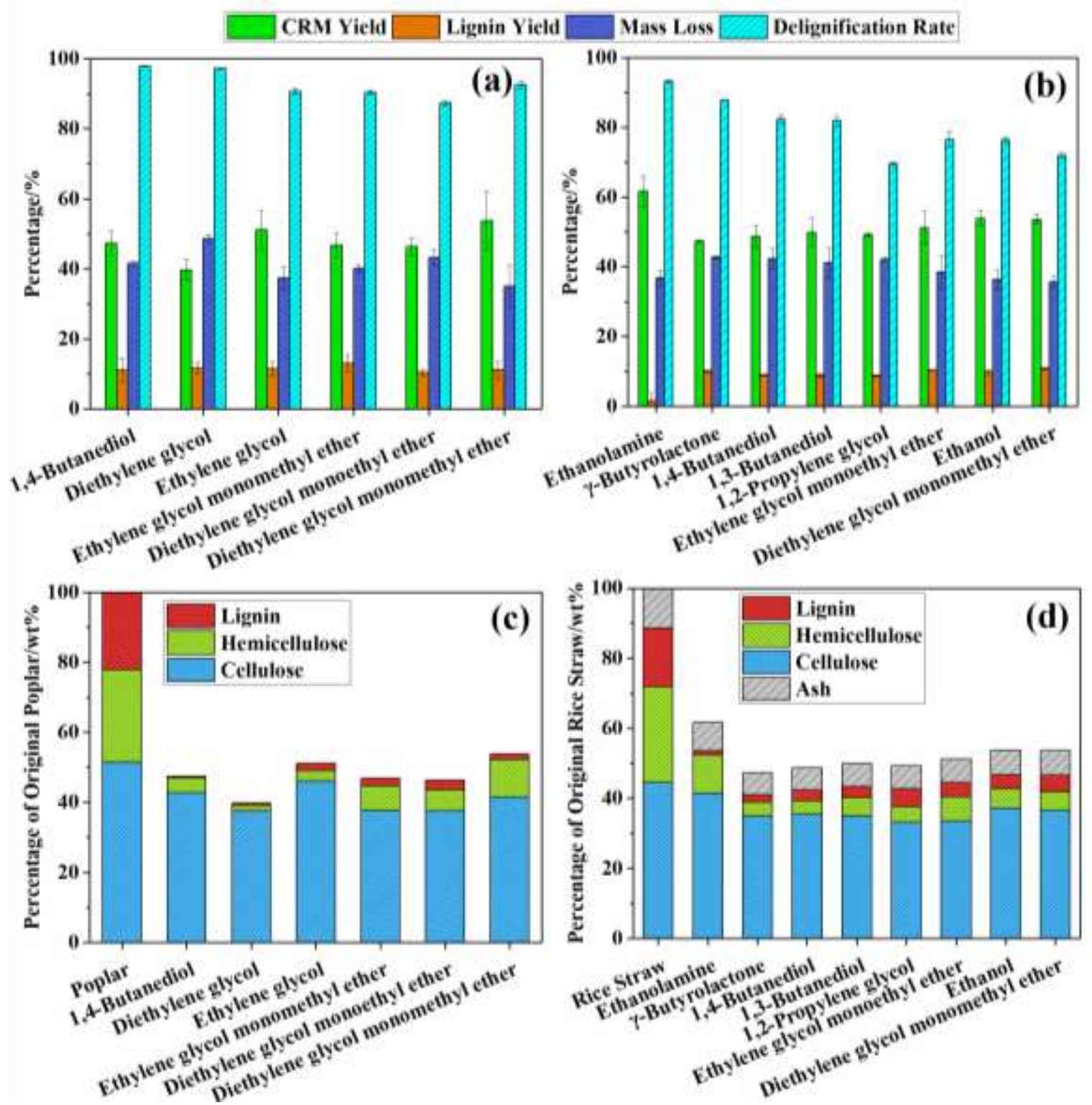

Fig. 1. Biomass fractionation results for different organosolv fractionation media: (a) component yields and delignification rates for poplar; (b) component yields and delignification rates for rice straw; (c) component contents of CRMs for poplar; (d) component contents of CRMs for rice straw. The groups are listed in the order of RED values of the fractionation media for lignin dissolution from lowest to highest.

Rice straw was treated with eight selected organic solvents as co-media to evaluate how they affected biomass fractionation. As compared to the poplar as the biomass source, more types of co-solvents with water were employed to fractionate rice straw, which included four alcohols (1,4-butanediol, 1,3-butanediol, 1,2-propylene glycol, and ethanol), two alcohol ethers (ethylene glycol monoethyl ether and diethylene glycol monomethyl ether), one lactone ( $\gamma$-butyrolactone), and one alkanolamine (ethanolamine). Organosolv fractionation with ethanolamine exhibited the highest delignification capacity, the highest delignification selectivity, and the highest CRM yield. However, the dissolved lignin in the ethanolamine-water fractionation medium was difficult to precipitate, which led to the 
lowest lignin yields. These observations may be related to the alkalinity of ethanolamine, which can facilitate the delignification process of lignocellulosics and the production of alkali lignin through the cleavage of lignin ether linkages and lignin-carbohydrate complexes (LCCs) (Zhao et al. 2018). Since the alkali lignin is water-soluble and can only be precipitated with the addition of strong acid, limited lignin was obtained by dilution with water addition to the spent fractionation liquor. Rodríguez et al. (2008) have reported that organosolv fractionation of rice straw with an $80 \%$ aqueous ethanolamine solution at $180{ }^{\circ} \mathrm{C}$ for $150 \mathrm{~min}$ afforded greater kappa number reductions than if the fractionation medium used ethylene glycol or diethylene glycol as a co-solvent. Additionally, organosolv fractionation of rice straw using $\gamma$-butyrolactone as a co-solvent, which can be produced from biomass conversion (Hu et al. 2016), afforded higher mass losses and higher cellulose purity in the CRM than other co-solvent. Similarly, $\gamma$-valerolactone, a homolog of $\gamma$ butyrolactone, has also showed high capacity on biomass conversion. The acid-catalyzed biomass conversion process using aqueous $\gamma$-valerolactone solution was able to produce soluble carbohydrates from corn stover, hardwood, and softwood at high yields of 70 to 90\% (Luterbacher et al. 2014). Moreover, $\gamma$-valerolactone has been demonstrated to fractionate wood to recover cellulose, sugar components from the hemicelluloses, and lignin. Organosolv fractionation conducting at $170{ }^{\circ} \mathrm{C}$ for $2 \mathrm{~h}$ resulted in a pulp yield of $40.3 \%$ with a high cellulose purity of $90.3 \%$ (Fang and Sixta 2015). This study indicated that $\gamma$-butyrolactone could be used as potential alternative to $\gamma$-valerolactone or other highboiling-point solvents in the organosolv fractionation process. The delignification efficiencies of the organosolv fractionation processes of rice straw decreased as lignin solubility decreased in the selected fractionation medium (i.e., RED value in Table 1).

\section{Correlations between Solubility Parameters of Co-Solvents and Delignification Efficiencies}

It is known that the properties of the organosolv fractionation solvent play an important role in biomass fractionation efficiency (Zhao et al. 2009). Researchers have made great efforts to investigate the influence of different types of co-solvents on biomass processing with organosolv fractionation (Li et al. 2016). Several studies suggested that the Hildebrand solubility parameters can be used to predict and optimize biomass fractionation with the organosolv process by adjusting the organic solvent concentration in water (Quesada-Medina et al. 2010; Ye et al. 2014). In the present study, correlations between Hansen solubility parameters of the selected fractionation media and the fractionation results were investigated. RED, an effective parameter in Hansen solubility theory (Hansen 2007), was used to investigate the relationships of biomass delignification with the organosolv fractionation medium used. As shown in Fig. 1, the increase of RED of solvent systems for lignin dissolution resulted in an increase of CRM yield and a decrease of mass loss. Meanwhile, lower delignification rate and higher residual lignin content in CRM could be obtained as the RED increased. This behavior is easily understood since the delignification process is always accompanied by the degradation of carbohydrates (Bajpai 2011). Hence, removal of hemicelluloses showed a similar trend as the delignification rate for all the organosolv processes examined (Fig. 1(c) and (d)). The noticeable influence of RED value of the co-solvent system on delignification performance indicated the potential correlation between solubility parameters and delignification. Nevertheless, the lignin yield for the processes of poplar and rice straw did not demonstrate a clear trend as the increase of RED. This may be attributed to the degradation of lignin during the process as reported previously (Ouyang et al. 2018). 


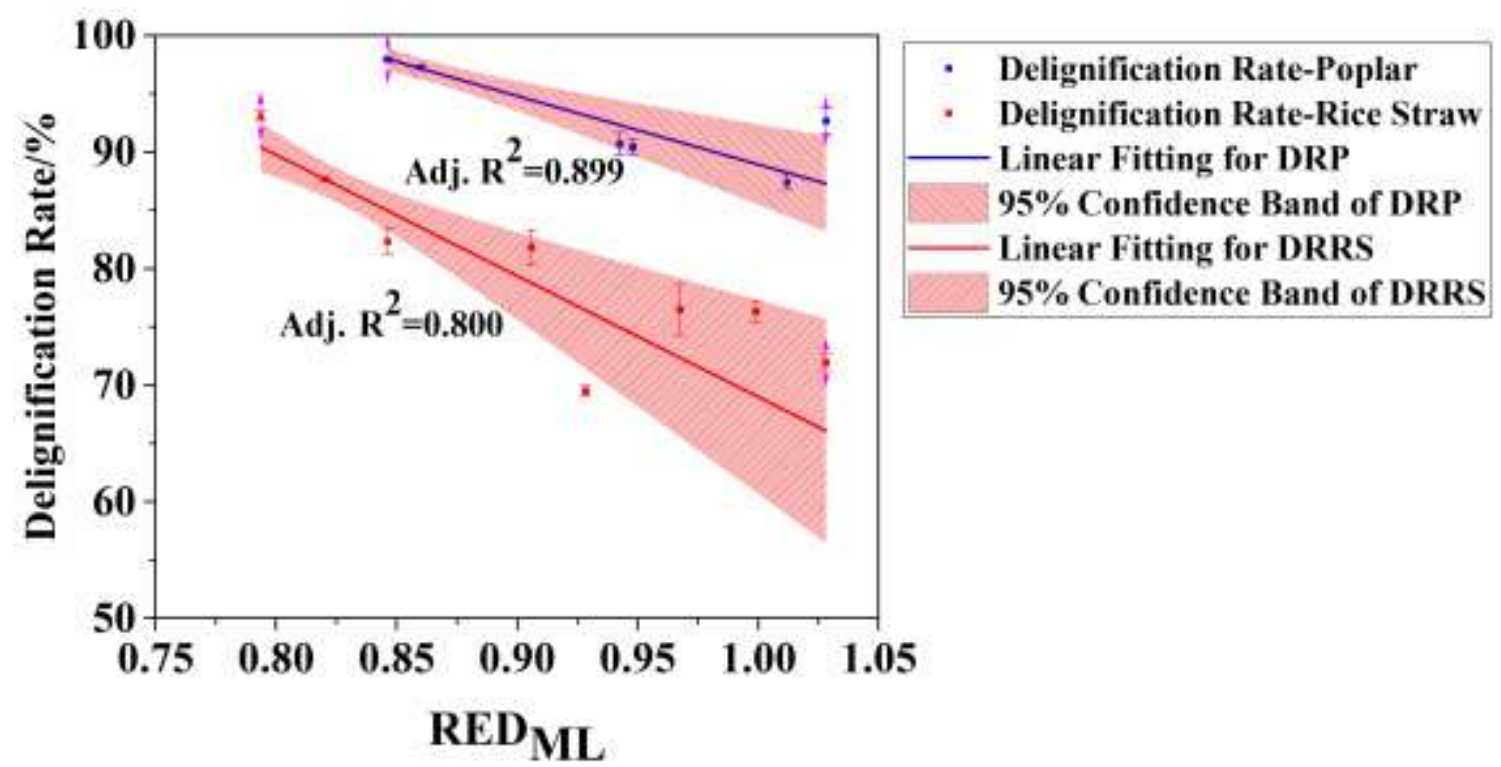

Fig. 2. Linear correlations between RED for lignin dissolution and the organosolv delignification rates for poplar and rice straw (DRP: delignification rate of poplar; and DRRS: delignification rate of rice straw processes)

Correlations between RED and delignification rates were further analyzed by linear regression analyses. The curves and corresponding adjusted coefficient of determination (Adj. $R^{2}$ ) for the regressions are summarized in Fig. 2. Interestingly, the RED of the cosolvent systems for lignin dissolution was closely correlated to biomass delignification in the organosolv process. The linear regression between these two factors resulted in a high Adj. $R^{2}$ of 0.899 and 0.800 for poplar and rice straw, respectively. Most data in the plot are located within the $95 \%$ confidence band, which indicated the close correlations between them. Notably, the regression for poplar organosolv fractionation showed a higher $R^{2}$ than that for rice straw. It was deduced that the correlation between REDs and delignification degree was reduced when increasing the type of co-solvent systems used in the organosolv fractionation processes. Additionally, as mentioned above, rice straw possesses higher ash content (mainly silica) than other biomasses, which increased its recalcitrance to pretreatment, and fractionation (or pulping). Thus, the less significant correlation between REDs and delignification degree should be also partially ascribed to high ash content in rice straw.

The Hildebrand solubility parameter for organic solvent with various concentrations has been shown to be closely related to delignification efficiencies in organosolv fractionation (Quesada-Medina et al. 2010; Ye et al. 2014; Weerachanchai et al. 2014). Nevertheless, it is widely accepted that Hildebrand solubility parameters are not applicable to polar solvent (or co-solvent) systems, since the parameters do not take into account the influences of hydrogen-bonding or polar interactions (Mannistu et al. 1997). Hansen's three-dimensional solubility theory takes into account the above interactions, along with dispersion interactions, with solute dissolution in a solvent or co-solvent system (Hansen 2007). Cheng et al. (2018) showed the potential of Hansen solubility parameter analysis with alcohol-based organosolv fractionation. The present study tried to investigate its application on the solvent selection for organosolv fractionation processes among multiple types of solvents (alcohols, alcohol ethers, lactones, and alkanolamine). These results indicated that Hansen solubility parameters can be used as basic information to 
screen multifarious solvents for the organosolv fractionation. Meanwhile, the increase of solvent type may lead to less close correlation between solubility parameters and delignification degree. Consequently, it is suggested that the prediction of delignification based on Hansen solubility parameters should be conducted among solvents of the same or analogical type.

Importantly, there is a need to further discuss the application of solubility parameters on the solvent screening and optimization of biomass fractionation processes. The organosolv fractionation process of biomass is often conducted in a combined system of biomass, solvent system and catalyst at elevated temperature and pressure. The delignification of biomass under this condition is extremely complicated, involving the depolymerization, dissolution and repolymerization of lignin (Pandey and Kim 2011). Any change on conditions and systems will result in different delignification efficiency. Herein, the study of biomass fractionation process was simplified to focus on the effects of the solvent system on the delignification efficiency, while other factors remained consistent. Therefore, the raw biomass and the catalyst must be optimized or determined before applying solubility parameter in the solvent screening and optimization of the biomass fractionation process.

At the same time, it is necessary to point out that the nature of the biomass feedstock itself also greatly influences the fractionation efficiency of the components. For example, the high ash content of rice straw in this study hindered the effective removal of lignin. Thus it is necessary to select a suitable catalyst to remove the ash and achieve a better delignification rate. In this context, the solubility parameter theory can be used as an auxiliary means to screen solvents for the biomass fractionation process after clarifying the nature of the raw biomass and the catalytic mechanism of catalyst.

\section{CONCLUSIONS}

1. RED of organic solvent-water systems for lignin dissolution was closely correlated to biomass delignification in the organosolv fractionation process, and adjusted coefficients of determination were 0.899 and 0.800 for poplar and rice straw, respectively.

2. Several organic solvents were employed in organosolv fractionation processes for the first time, and the processes using these solvents showed promising efficiencies for biomass delignification with a delignification rate of more than $90 \%$.

3. The organosolv systems, those combined twelve organic solvents with water and AIL respectively, can fractionate poplar and rice straw into CRM (40 to $62 \%$ of the original biomass) and lignin (9 to $14 \%$ of the original biomass) and simultaneously convert most of hemicellulose.

\section{ACKNOWLEDGMENTS}

The authors are grateful for the support of the Natural Science Foundation of Guangxi (2016GXNSFCA380025), the National Natural Science Foundation of China (31760183), the Project Funded by China Postdoctoral Science Foundation 
(2017M620361) and the Scientific Research Foundation of Guangxi University (XGZ150513).

\section{REFERENCES CITED}

Alok, S., Ruchi, A., Samarthya, B., Parthapratim, D., and J., R. A. (2018). "Rice straw as a feedstock for biofuels: Availability, recalcitrance, and chemical properties," Biofuel. Bioprod. Bior. 12(1), 83-107. DOI: 10.1002/bbb.1818

Amiri, H., Karimi, K., and Zilouei, H. (2014). "Organosolv pretreatment of rice straw for efficient acetone, butanol, and ethanol production," Bioresource Technol. 152, 450456. DOI: 10.1016/j.biortech.2013.11.038

Bajpai, P. (2011). Environmentally Friendly Production of Pulp and Paper, John Wiley \& Sons, Hoboken, NJ.

Barton, A. F. M. (1975). “Solubility parameters," Chem. Rev. 75(6), 731-753. DOI: $10.1021 / \mathrm{cr} 60298 \mathrm{a} 003$

Benazzouz, A., Moity, L., Pierlot, C., Sergent, M., Molinier, V., and Aubry, J.-M. (2013). "Selection of a greener set of solvents evenly spread in the Hansen space by spacefilling design," Ind. Eng. Chem. Res. 52(47), 16585-16597. DOI: 10.1021/ie402410w

Cantarella, M., Cantarella, L., Gallifuoco, A., Spera, A., and Alfani, F. (2004).

"Comparison of different detoxification methods for steam-exploded poplar wood as a substrate for the bioproduction of ethanol in SHF and SSF," Process Biochem. 39(11), 1533-1542. DOI: 10.1016/S0032-9592(03)00285-1

Cheng, F., Wang, H., Chatel, G., Gurau, G., and Rogers, R. D. (2014). "Facile pulping of lignocellulosic biomass using choline acetate," Bioresource Technol. 164, 394-401. DOI: 10.1016/j.biortech.2014.05.016

Cheng, F., Zhao, X., and Hu, Y. (2018). "Lignocellulosic biomass delignification using aqueous alcohol solutions with the catalysis of acidic ionic liquids: A comparison study of solvents," Bioresource Technol. 249, 969-975. DOI: 10.1016/j.biortech.2017.10.089

Datta, R. (1981). "Acidogenic fermentation of lignocellulose-acid yield and conversion of components," Biotechnol. Bioeng. 23(9), 2167-2170. DOI: 10.1002/bit.260230921

Dence, C.W. (1992). "The determination of lignin," in: S. Y. Lin and C. W. Dence (eds.) Methods in Lignin Chemistry, Springer, Berlin, Germany, pp. 33-61.

Fang, W., and Sixta, H. (2015). "Advanced biorefinery based on the fractionation of biomass in $\gamma$-valerolactone and water," ChemSusChem 8(1), 73-76. DOI: $10.1002 /$ cssc. 201402821

GB/T 742-2008. Fibrous raw material, pulp, paper and board-Determination of ash, Chinese National Standards, 2008.

Hansen, C. M. (2007). Hansen Solubility Parameters: A User's Handbook, CRC Press, Boca Raton, FL, USA.

Haysom, M. B., and Ostatek-Boczynski, Z. A. (2006). "Rapid, wet oxidation procedure for the estimation of silicon in plant tissue," Commun. Soil Sci. Plant Anal. 37(15-20), 2299-2306. DOI: 10.1080/00103620600819420

Hildebrand, J. H., and Scott, R. L. (1950). The Solubility of Nonelectrolytes ( $3^{\text {rd }}$ Ed.), Reinhold Publishing Corporation, New York, NY.

Hou, X.-D., Li, A.-L., Lin, K.-P., Wang, Y.-Y., Kuang, Z.-Y., and Cao, S.-L. (2018). "Insight into the structure-function relationships of deep eutectic solvents during rice 
straw pretreatment," Bioresource Technol. 249, 261-267. DOI:

10.1016/j.biortech.2017.10.019

Hu, E., Gao, F., Xin, Y., Jia, H., Li, K., Hu, J., and Feng, Z. (2015). “Concentration- and flux-based ozone dose-response relationships for five poplar clones grown in North China," Environ. Pollut. 207, 21-30. DOI: 10.1016/j.envpol.2015.08.034

Hu, Q., Fan, G., Yang, L., Cao, X., Zhang, P., Wang, B., and Li, F. (2016). "A gas-phase coupling process for simultaneous production of $\gamma$-butyrolactone and furfuryl alcohol without external hydrogen over bifunctional base-metal heterogeneous catalysts," Green Chem. 18(8), 2317-2322. DOI: 10.1039/c5gc02924d

Jahan, M. S., Sutradhar, S., Rahman, M. M., and Quaiyyum, M. A. (2015). "Fractionation of rice straw for producing dissolving pulp in biorefinery concept," Nord. Pulp Paper Res. J. 30(4), 562-567.

Kangas, H., Liitiä, T., Rovio, S., Ohra-aho, T., Heikkinen, H., Tamminen, T., and Poppius-Levlin, K. (2015). "Characterization of dissolved lignins from acetic acid Lignofibre (LGF) organosolv pulping and discussion of its delignification mechanisms," Holzforschung 69(3), 247-256. DOI: 10.1515/hf-2014-0070

Li, M.-F., Yang, S., and Sun, R.-C. (2016). "Recent advances in alcohol and organic acid fractionation of lignocellulosic biomass," Bioresource Technol. 200, 971-980. DOI: 10.1016/j.biortech.2015.10.004

Luterbacher, J. S., Rand, J. M., Alonso, D. M., Han, J., Youngquist, J. T., Maravelias, C. T., Pfleger, B. F., and Dumesic, J. A. (2014). "Nonenzymatic Sugar Production from Biomass Using Biomass-Derived $\gamma$-Valerolactone," Science 343(6168), 277-280. DOI: $10.1126 /$ science. 1246748

Lv, H., Yan, L., Zhang, M., Geng, Z., Ren, M., and Sun, Y. (2013). "Influence of supercritical $\mathrm{CO}_{2}$ pretreatment of corn stover with ethanol-water as co-solvent on lignin degradation," Chem. Eng. Technol. 36(11), 1899-1906. DOI: 10.1002/ceat.201300183

Mannistu, K. D., Yarranton, H. W., and Masliyah, J. H. (1997). "Solubility modeling of asphaltenes in organic solvents," Energ. Fuel. 11(3), 615-622. DOI: $10.1021 / \mathrm{ef} 9601879$

Oliet, M., García, J., Rodríguez, F., and Gilarrranz, M. A. (2002). "Solvent effects in autocatalyzed alcohol-water pulping: Comparative study between ethanol and methanol as delignifying agents," Chem. Eng. J. 87(2), 157-162. DOI: 10.1016/S1385-8947(01)00213-3

Ouyang, T., Wang, L., Cheng, F., Hu, Y., and Zhao, X. (2018). "Lignocellulose fractionation and lignin depolymerization using glycerol and acidic ionic liquids: Identification of the main products by GC-MS," BioResources 13(2), 3880-3891. DOI: 10.15376/biores. 13.2.3880-3891

Pandey, M. P., and Kim, C. S. (2011). "Lignin depolymerization and conversion: A review of thermochemical methods," Chem. Eng. Technol. 34(1), 29-41. DOI: 10.1002/ceat.201000270

Quang, L. H., Anna, Z., Juha-Pekka, P., Marina, S., Ville, A., and Herbert, S. (2016). "Solubility of organosolv lignin in $\gamma$-valerolactone/water binary mixtures," ChemSusChem 9(20), 2939-2947. DOI: 10.1002/cssc.201600655

Quesada-Medina, J., López-Cremades, F. J., and Olivares-Carrillo, P. (2010). "Organosolv extraction of lignin from hydrolyzed almond shells and application of the $\delta$-value theory," Bioresource Technol. 101(21), 8252-8260. DOI:

10.1016/j.biortech.2010.06.011 
Rodríguez, A., Serrano, L., Moral, A., and Jiménez, L. (2008). "Pulping of rice straw with high-boiling point organosolv solvents," Biochem. Eng. J. 42(3), 243-247. DOI: 10.1016/j.bej.2008.07.001

Romaní, A., Garrote, G., López, F., and Parajó, J. C. (2011). “Eucalyptus globulus wood fractionation by autohydrolysis and organosolv delignification," Bioresource Technol. 102(10), 5896-5904. DOI: 10.1016/j.biortech.2011.02.070

Santo, M. E., Rezende, C. A., Bernardinelli, O. D., Pereira, N., Curvelo, A. A. S., deAzevedo, E. R., Guimarães, F. E. G., and Polikarpov, I. (2018). "Structural and compositional changes in sugarcane bagasse subjected to hydrothermal and organosolv pretreatments and their impacts on enzymatic hydrolysis," Ind. Crop. Prod. 113, 64-74. DOI: 10.1016/j.indcrop.2018.01.014

Schrems, M., Liebner, F., Betz, M., Zeilinger, M., Böhmdorfer, S., Rosenau, T., and Potthast, A. (2012). "Understanding the impact of supercritical carbon dioxide on the delignification mechanism during organosolv pulping: A model compound study," $J$. Wood Chem. Technol. 32(3), 225-237. DOI: 10.1080/02773813.2011.652856

Sun, R. C., Tomkinson, J., Ma, P. L., and Liang, S. F. (2000). "Comparative study of hemicelluloses from rice straw by alkali and hydrogen peroxide treatments," Carbohydr. Polym. 42(2), 111-122. DOI: 10.1016/S0144-8617(99)00136-8

Weerachanchai, P., Kwak, S. K., and Lee, J.-M. (2014). "Effects of solubility properties of solvents and biomass on biomass pretreatment," Bioresource Technol. 170, 160166. DOI: 10.1016/j.biortech.2014.07.057

Xi, J., Ding, D., Shao, Y., Liu, X., Lu, G., and Wang, Y. (2014). "Production of ethylene glycol and its monoether derivative from cellulose," ACS Sust. Chem. Eng. 2(10), 2355-2362. DOI: $10.1021 / \mathrm{sc} 500380 \mathrm{c}$

Ye, Y., Liu, Y., and Chang, J. (2014). "Application of solubility parameter theory to organosolv extraction of lignin from enzymatically hydrolyzed cornstalks," BioResources 9(2), 3417-3427.

Zhao, X., Cheng, K., and Liu, D. (2009). "Organosolv pretreatment of lignocellulosic biomass for enzymatic hydrolysis," Appl. Microbiol. Biotechnol. 82(5), 815-827. DOI: $10.1007 / \mathrm{s} 00253-009-1883-1$

Zhao, Z., Chen, X., Ali, M. F., Abdeltawab, A. A., Yakout, S. M., and Yu, G. (2018). "Pretreatment of wheat straw using basic ethanolamine-based deep eutectic solvents for improving enzymatic hydrolysis," Bioresource Technol. 263, 325-333. DOI: 10.1016/j.biortech.2018.05.016

Zhu, J. Y., Pan, X., and Zalesny, R. S. (2010). "Pretreatment of woody biomass for biofuel production: energy efficiency, technologies, and recalcitrance," Appl. Microbiol. Biotechnol. 87(3), 847-857. DOI: 10.1007/s00253-010-2654-8

Article submitted: July 24, 2018; Peer review completed: September 17, 2018; Revised version received: October 16, 2018; Accepted: November 22, 2018; Published:

November 28, 2018.

DOI: $10.15376 /$ biores.14.1.486-499 


\section{APPENDIX}

\section{Supplementary Material}

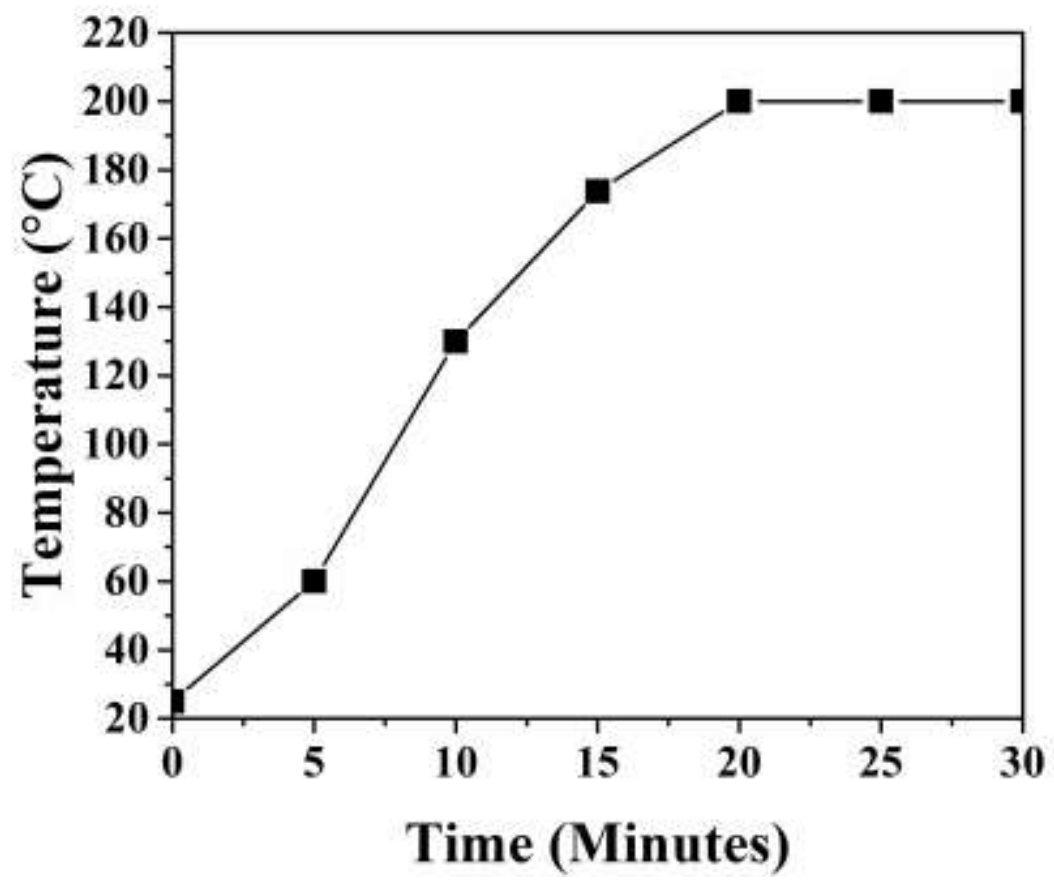

Fig. S1. Typical temperature profile during the fractionation process 